\title{
Moment of inertia just above $\lambda$ point
}

\author{
Shun-ihiro Koh \\ Physics Division, Faculty of Education, Kochi University, Akebono-cho, \\ 2-5-1,Kochi, 780, Japan
}

The moment of inertia of a quantum liquid of a bulk sample is examined just above $\lambda$ point. Although the macroscopic condensate does not yet appear above $T_{\lambda}$, under the strong influence of Bose statistics, the coherent manybody wave function grows to an intermediate size between a macroscopic and a microscopic one as a thermal equilibrium state, which must affect rotational properties of a liquid. Beginning with the bosons without the condensate, we make a perturbation calculation of its susceptibility with respect to the repulsive interaction. By taking peculiar diagrams obeying Bose statistics, we examine how, with decreasing temperature, the growth of the coherent wave function gradually changes the rotational behavior of a liquid, with a result that the moment of inertia slightly decreases prior to $T_{\lambda}$. This means that at the vicinity of $T_{\lambda}$ the superfluid density defined in the mechanical phenomena does not always agree with that defined in thermodynamics. Its implication to the recent discovery of nonclassial moment of inertia in a solid helium 4 is discussed.

PACS numbers: 67.80.-s, 67.80 Mg, 67.40.-w, 05.30 Jp

\section{INTRODUCTION}

The change of the moment of inertia is a remarkable phenomenon characterizing superfluidity. In a liquid helium 4 just above $T_{\lambda}$, it is known that the short-lived coherent wave functions appear owing to thermal fluctuations, which cause anomalies accompanying the phase transition. Superfluidity, however, is not caused by thermal fluctuations, because it requires a specific direction of motions continuing during the characteristic time of macroscopic measurements. The moment of inertia $I_{z}=L / \Omega$ is the transverse response of the system to the rotation of a container. ${ }^{1}$ The perturbation energy due 


\section{S.Koh}

to the external field $\boldsymbol{v}_{d}(\boldsymbol{r})=\boldsymbol{v} \times \boldsymbol{r}$ is given by

$$
-\boldsymbol{\Omega} \cdot \boldsymbol{L}=-\int \boldsymbol{v}_{d}(\boldsymbol{r}) \cdot \boldsymbol{J}(\boldsymbol{r}) d \boldsymbol{r},
$$

where the mass current $\boldsymbol{J}(\boldsymbol{r})$ is determined by the transverse susceptibility

$$
\boldsymbol{J}(\boldsymbol{r})=\left[\lim _{q \rightarrow 0} \chi^{T}(q, 0)\right] \boldsymbol{v}_{d}(\boldsymbol{r}) .
$$

On the other hand, the classical moment of inertia is given by

$$
I_{z}^{c}=\chi^{L}(0,0) \int_{V}\left(x^{2}+y^{2}\right) d \mathbf{r},
$$

where $\chi^{L}(0,0)=m n$ is the longitudinal susceptibility ( $m$ and $n$ is a mass and a number density of the boson, respectively). Generally, the susceptibility is decomposed to the transverse and longitudinal parts

$$
\begin{aligned}
\chi_{\mu \nu}(q, \omega) & =\delta_{\mu \nu} \chi^{T}(q, \omega)+q_{\mu} q_{\nu}\left(\frac{\chi^{L}(q, \omega)-\chi^{T}(q, \omega)}{q^{2}}\right) \\
& \equiv \delta_{\mu \nu} \chi^{T}+\hat{\chi}_{\mu \nu}(q, \omega) .
\end{aligned}
$$

Comparing Eqs.(1), (2) and (3), and using a factor $\hat{\chi}_{\mu \nu}$ proportional to $q_{\mu} q_{\nu}$ in Eq.(4), one obtains a general form of the moment of inertia

$$
I_{z}=I_{z}^{c}-\lim _{q \rightarrow 0}\left[\frac{q^{2}}{q_{\mu} q_{\nu}} \hat{\chi}_{\mu \nu}(q, 0)\right] \int_{V}\left(x^{2}+y^{2}\right) d \mathbf{r} .
$$

In the normal phase, $\chi^{L}(0,0)=\chi^{T}(0,0)$, hence $\hat{\chi}_{\mu \nu}(0,0)=0$ in Eq.(5). Thus $I_{z}$ agrees with $I_{z}^{c}$. Only when $\hat{\chi}_{\mu \nu}$ diverges as $q^{-2}$ at $q \rightarrow 0, I_{z}$ becomes different from $I_{z}^{c}$. The coefficient of $\hat{\chi}_{\mu \nu}$ corresponds to the superfluid density.

Superfluidity (not fluctuations accompanying it) is normally assumed to abruptly occur when the system reaches $\lambda$ point. (The decrease of the moment of inertia is a typical example.) Generally, however, the overall transformation of the system occurs more or less continuously. In Sec.2, we will show that the interplay between Bose statistics and the repulsive interaction makes the moment of inertia slightly decrease just above $T_{\lambda}$ in which the coherent wave functions are large but not yet macroscopic. ${ }^{2}$

This phenomenon shares a common feature with the recent discovery in a solid helium 4 that a slight decrease of moment of inertia occurs in a system in which the macroscopic condensate is not expected. In Sec.3, we discuss a solid helium 4 from this viewpoint. 


\section{Moment of inertia just above $\lambda$ point}

\section{FORMALISM}

A liquid helium 4 is well described using the following short-range repulsive boson model

$$
H=\sum_{p} \epsilon(p) \Phi_{p}^{\dagger} \Phi_{p}+U \sum_{p, p^{\prime}} \sum_{q} \Phi_{p-q}^{\dagger} \Phi_{p^{\prime}+q}^{\dagger} \Phi_{p^{\prime}} \Phi_{p} .
$$

(In a solid helium 4 at low temperature, the lattice vacancy changes its position more frequently than that in an ordinary crystal, which appears as the zero-point motion of helium 4 atoms consisting of a crystal. Although it is not experimentally confirmed that such a "zero-point vacancy" (ZPV) moves freely in a crystal, it is likely that something movable has the same hamiltonian as Eq.(6).)

$\chi_{\mu \nu}$ in Eq.(4) is defined under the hamiltonian Eq.(6) as follows,

$$
\chi_{\mu \nu}\left(q, \omega_{n}\right)=\frac{1}{V} \int_{0}^{\beta} d \tau \exp \left(i \omega_{n} \tau\right)<G\left|T_{\tau} J_{\mu}(q, \tau) J_{\nu}(q, 0)\right| G>,
$$

where

$$
J_{\mu}(q, \tau)=\sum_{p, n}\left(p+\frac{q}{2}\right)_{\mu} \Phi_{p}^{\dagger} \Phi_{p+q} e^{i \omega_{n} \tau} .
$$

In an ideal Bose gas, $\hat{\chi}_{\mu \nu}$ is given by

$$
\hat{\chi}_{\mu \nu}(q, \omega)=-\frac{q_{\mu} q_{\nu}}{4} \frac{1}{V} \sum_{p} \frac{f(\epsilon(p))-f(\epsilon(p+q))}{\omega+\epsilon(p)-\epsilon(p+q)} .
$$

At $T<T_{\lambda}$, the Bose distribution $f(\epsilon)$ is a macroscopic number $n_{c}$ for $p=0$ or $p=-q$, and nearly zero for other momentums. Hence, at $T<T_{\lambda}$,

$$
\hat{\chi}_{\mu \nu}(q, 0)=m n_{c} \frac{q_{\mu} q_{\nu}}{q^{2}} .
$$

Using Eq.(10) in Eq.(5), one obtains the well-known nonclassical $I_{z}$. This means that the macroscopic condensate is a necessary condition for the nonclassical moment of inertia in an ideal Bose gas.

For the repulsive boson system like Eq.(6), we must take into account of the repulsive interaction $H_{I}$ as follows,

$$
\begin{aligned}
& <G\left|T_{\tau} J_{\mu}(x, \tau) J_{\nu}(0,0)\right| G> \\
& \quad=\frac{<0\left|T_{\tau} \hat{J}_{\mu}(x, \tau) \hat{J}_{\nu}(0,0) \exp \left[-\int_{0}^{\beta} d \tau \hat{H}_{I}(\tau)\right]\right| 0>}{<0\left|\exp \left[-\int_{0}^{\beta} d \tau \hat{H}_{I}(\tau)\right]\right| 0>} .
\end{aligned}
$$

At the vicinity of $T_{\lambda}$ in the normal phase, the coherent wave function has not yet grown to a macroscopic size, but already to a large one. Hence, bosons 


\section{S.Koh}

in $J_{\mu} J_{\nu}$ and $H_{I}$ in Eq.(11) forms a coherent wave function obeying Bose statistics. A particle exchange between $J_{\mu} J_{\nu}$ and $H_{I}$ leads to the following next-order $\chi_{\mu \nu}$ ( $\Sigma$ is a self energy owing to the repulsion. See Fig.3 of Ref.2)

$$
\chi_{\mu \nu}(q, \omega)=U \frac{1}{V} \sum_{p}\left(p+\frac{q}{2}\right)_{\mu}\left(p+\frac{q}{2}\right)_{\nu}\left[-\frac{f(\epsilon(p)+\Sigma)-f(\epsilon(p+q)+\Sigma)}{\omega+\epsilon(p)-\epsilon(p+q)}\right]^{2} .
$$

With decreasing temperature, the coherent wave function grows to a large size, and the interchange of bosons due to Bose statistics occurs many times. Hence, one cannot ignore the higher-order terms which are significant in the larger coherent wave function. Among many bosons with various momentums, bosons with zero momentum play a key role. Hence, we obtain the following form of $\hat{\chi}_{\mu \nu}$,

$$
\hat{\chi}_{\mu \nu}(q, 0)=\frac{q_{\mu} q_{\nu}}{2} \frac{1}{V} \sum_{n=0}^{\infty} U^{n} F_{\beta}(q)^{n+1},
$$

where $F_{\beta}(q)$ is a positive monotonously decreasing function of $q^{2}$,

$$
F_{\beta}(q)=\frac{(\exp (\beta[\Sigma-\mu]-1))^{-1}-(\exp (\beta[\epsilon(q)+\Sigma-\mu])-1)^{-1}}{\epsilon(q)} .
$$

When $T \gg T_{\lambda}$, a small $F_{\beta}(q)$ guarantees the convergence of Eq.(13), with a result that

$$
\hat{\chi}_{\mu \nu}(q, 0)=\frac{q_{\mu} q_{\nu}}{2} \frac{1}{V} \frac{F_{\beta}(q)}{1-U F_{\beta}(q)} .
$$

With decreasing temperature, however, $F_{\beta}(q)$ gradually grows, and the convergence of Eq.(13) becomes questionable. Its behavior around $q^{2}=0$ is given by

$$
F_{\beta}(q)=\frac{\beta}{4 \sinh ^{2}\left(\frac{\mid \beta[\mu(T)-\Sigma]}{2}\right)}\left[1-\frac{\beta}{2} \frac{1}{\tanh \left(\frac{|\beta[\mu(T)-\Sigma]|}{2}\right)} \frac{q^{2}}{2 m}+\cdots\right] .
$$

When the following condition is satisfied,

$$
U \beta=4 \sinh ^{2}\left(\frac{\beta[\mu-\Sigma]}{2}\right),
$$

the denominator $1-U F_{\beta}(q)$ in Eq.(15) gets to begin with $q^{2}$. Hence, $\hat{\chi}_{\mu \nu}(q, 0)$ has $q^{-2}$ singularity

$$
\hat{\chi}_{\mu \nu}(q, 0)=\frac{1}{V} \frac{m}{\sinh |\beta[\mu-\Sigma]|} \frac{q_{\mu} q_{\nu}}{q^{2}} .
$$




\section{Moment of inertia just above $\lambda$ point}

At $T_{\lambda}, \mu=\Sigma$ is satisfied. With a nonzero $U$, Eq.(17) is satisfied at $T_{o n}$ higher than $T_{\lambda}$. Using Eq.(18) in Eq.(5), we know that with decreasing temperature, the moment of inertia obtains a nonclassical value at $T_{\text {on }}$ prior to $T_{\lambda}$

$$
I_{z}\left(T_{o n}\right)=I_{z}^{c l}\left(1-c\left(T_{o n}\right) \frac{n_{0}\left(T_{o n}\right)}{n}\right) \equiv I_{z}^{c l}\left(1-\frac{\hat{\rho}_{s}\left(T_{o n}\right)}{\rho}\right),
$$

where $n_{0}(T)=V^{-1} /(\exp (-\beta[\mu(T)-\Sigma])-1)$ is the number density of $p=0$ bosons, and $c(T)=2 /(\exp (\beta|\mu(T)-\Sigma|)+1)$ is a Fermi-distributionlike coefficient. $\hat{\rho}_{s}(T) \equiv m c(T) n_{0}(T)$ is the superfluid density defined in the mechanical phenomena such as the rotation, which is slightly different from the conventional $\rho_{s}(T)$ defined in thermodynamics. In the theoretical limit $V \rightarrow \infty, n_{0}(T)$ is normally regarded to be zero. In real system, however, its magnitude must be estimated by experiments. When one views previous experiments of a liquid helium 4 from this point, one notes, in the data by Hess and Fairbank, ${ }^{3}$ an experimental sign suggesting a slight decrease of $I_{z}$ of a bulk liquid helium 4 just above $T_{\lambda}$ (see Ref.2).

\section{IMPLICATION TO A SOLID HELIUM 4}

Solid helium 4 has been termed a quantum crystal. On introduction of defects, it displays several anomalies not observed in classical crystals. Vacancies can move about unusually rapidly. Hence, it is natural to expect that the quantum-mechanical fluctuation delocalizes vacancies at a sufficiently low temperature (ZPV). ${ }^{4}$ In such a 'supersolid state', nonclassical rotational properties are expected. ${ }^{5}$ A number of experiments have been made to search for anomalies in thermodynamical or mechanical properties

, but ended with null results. ${ }^{6}$ Recently, an abrupt drop in the moment of inertia was found in the torsional oscillation measurements on solid helium 4 confined in a porous media, and on a bulk solid helium $4 .^{7}$ This discovery raises a question why other measurements until now have found null results, and leads us to reconsider the definition of superfluidity and that of solids.

Anomalous behaviors of the system are normally attributed to the Bose condensate; in other words, off-diagonal long-range order (ODLRO). The question whether the Bose condensate is a necessary condition for superfluidity has been discussed in terms of academic interest. For solid helium 4, however, this question is not an academic but a practical one.

A fundamental feature of crystals lies in their periodicity in density; that is, diagonal long-range order (DLRO). One has to face a serious question whether crystals remain stable while showing superfluidity that violates their periodicity. Since vacancies are defects on the crystal lattice, it is unlikely 


\section{S.Koh}

that crystals with a macroscopic number of defects remain stable. Rather, in light of the definition of solids, a more natural idea is that the number of ZPV is large, but not macroscopic. Hence, a direction to be explored is the possibility that under the influence of Bose statistics a large but microscopic number of ZPV's create the non-classical rotational inertia without ODLRO. The conclusion in Sec.2 is along this line of thought. Something movable in a solid helium 4 forms a large but microscopic wave function, and slightly decreases the moment of inertia.

Recent energy calculation is not in favor of the existence of ZPV as a freely moving boson. ${ }^{8}$ Crystals are often split into domains. If interfaces show a non-classical behavior, a relatively small number of particles consisting of interfaces may influence a macroscopic number of particles. ${ }^{9}$ Specifically, if interfaces do not transmit the rotation from the outer region to the inner one, it decouples the inner region from the rotation of a container, thus leading to a slight decrease of the moment of inertia of a sample. Such a non-classical behavior must have an origin in Bose statistics. In this case, Eq.(6) is not applied to a point-like object, but must be applied to a two-dimensionally extended object like an interface.

Superfluidity is a complex of phenomena, and therefore has some different definitions. Since these phenomena are observed to occur together in most materials, it is difficult to elucidate the exact nature of the relationships between these definitions. In a solid helium 4, there is no reason to expect that all definitions of superfluidity should occur, and we must view some 'null results' in earlier mechanical measurements from this point. We can regard a solid helium 4, together with a helium 4 film, as appropriate material for examining the relationship between the different definitions of superfluidity.

\section{REFERENCES}

1. As a review, P.Nozieres, in Quantum Fluids (ed by D.E.Brewer), 1 (North Holland, Amsterdam, 1966), G.Baym, in Mathematical methods in Solid State and Superfluid Theory (ed by R.C.Clark and G.H.Derrick), 121 (Oliver and Boyd, Edingburgh, 1969).

2. S.Koh, Phys. Rev. B.74, 054501 (2006).

3. G.B.Hess and W.M.Fairbank, Phys. Rev. 19, 216(1967)

4. A.F.Andreef and I.M.Lifshitz, Zh.Eksp.teor.Fiz. 56, 2057(1969), [Sov. Phys. JETP. 29, 1107(1969)].

5. A.J.Leggett, Phys.Rev. Lett.25,1543(1970).

6. As a review, M.W.Meisel, Physica. B178, 121(1992).

7. E.Kim and M.H.W.Chan, Nature. 427, 225(2004), Science. 305, 1941(2004).

8. D.M.Ceperley and B.Bernu, Phys.Rev. Lett.93,155303(2004).

9. N.Prokof'ev and B.Svistunov, Phys.Rev. Lett.94,155302(2005). 